Title: Updates to Pediatric Hypertension Guidelines: Influence on Classification of High Blood Pressure in Children and Adolescents

Short title: Pediatric hypertension guideline

Total number of supplementary tables: 6

Total number of supplementary figures: 1

Table S1. Demographic characteristics of Chinese children and adolescents by sex (n $=50,336)$

Table S2. Differences in HBP prevalence among children and adolescents using the Fourth Report and CPG definitions

Table S3. The odds ratios of HBP with BMI groups and height groups in both sexes of two age groups using CPG and the Fourth Report definitions

Table S4. The interaction of odds ratios of HBP with BMI and height in different height groups and BMI groups in both sexes of two age groups using CPG and the Fourth Report definitions

Table S5. The prevalence of HBP in different height and BMI groups in both sexes of two age groups using the CPG and Fourth Report definitions

Table S6. Height Z-Score of children and adolescents aged 6-17 years

\title{
Figure legends:
}

Figure S1. The differences of systolic BP and diastolic BP reference values (P95) between the CPG and the Fourth Report across age groups in boys and girls 
Table S1. Demographic characteristics of Chinese children and adolescents by sex $(n=50,336)$

\begin{tabular}{|c|c|c|c|c|c|c|c|c|c|}
\hline \multirow{2}{*}{$\begin{array}{l}\text { Age, } \\
\text { years }\end{array}$} & \multirow{2}{*}{$\mathrm{N}$} & \multirow{2}{*}{$\mathrm{SBP}, \mathrm{mmHg}$} & \multirow{2}{*}{ DBP, $\mathrm{mmHg}$} & \multirow{2}{*}{ Height, cm } & \multirow{2}{*}{ Weight, kg } & \multirow{2}{*}{ BMI, $\mathrm{kg} / \mathrm{m}^{2}$} & \multicolumn{3}{|c|}{ BMI group } \\
\hline & & & & & & & Thinness & Overweight & Obesity \\
\hline \multicolumn{10}{|l|}{ Boys } \\
\hline 6 & 2,277 & $96.0 \pm 10.3$ & $61.1 \pm 8.4$ & $121.4 \pm 5.4$ & $24.5 \pm 5.4$ & $16.5 \pm 2.8$ & $254(11.2)$ & $314(13.8)$ & $223(9.8)$ \\
\hline 7 & 3,095 & $98.9 \pm 10.4$ & $63.0 \pm 8.2$ & $127.1 \pm 5.8$ & $27.3 \pm 6.0$ & $16.8 \pm 2.8$ & $290(9.4)$ & $465(15.0)$ & $313(10.1)$ \\
\hline 8 & 3,216 & $101.2 \pm 10.7$ & $65.0 \pm 8.2$ & $132.5 \pm 6.0$ & $31.0 \pm 7.7$ & $17.5 \pm 3.3$ & $254(7.9)$ & $556(17.3)$ & $371(11.5)$ \\
\hline 9 & 3,168 & $103.6 \pm 11.2$ & $66.0 \pm 8.4$ & $137.5 \pm 6.5$ & $34.6 \pm 8.8$ & $18.1 \pm 3.5$ & $231(7.3)$ & $629(19.9)$ & $370(11.7)$ \\
\hline 10 & 2,877 & $105.6 \pm 11.3$ & $67.2 \pm 8.7$ & $142.8 \pm 7.1$ & $38.9 \pm 10.3$ & $18.9 \pm 3.7$ & $203(7.1)$ & $675(23.5)$ & $294(10.2)$ \\
\hline 11 & 874 & $107.1 \pm 11.1$ & $67.7 \pm 8.2$ & $146.4 \pm 7.4$ & $41.5 \pm 11.2$ & $19.1 \pm 4.0$ & $76(8.7)$ & $184(21.1)$ & $78(8.9)$ \\
\hline 12 & 2,405 & $107.8 \pm 12.1$ & $67.9 \pm 8.9$ & $156.6 \pm 8.6$ & $49.4 \pm 13.2$ & $20.0 \pm 4.1$ & $163(6.8)$ & $505(21.0)$ & $233(9.7)$ \\
\hline 13 & 2,799 & $110.9 \pm 11.3$ & $68.5 \pm 8.5$ & $162.1 \pm 8.4$ & $53.9 \pm 13.9$ & $20.4 \pm 4.1$ & $195(7.0)$ & $531(19.0)$ & $239(8.5)$ \\
\hline 14 & 879 & $112.5 \pm 11.5$ & $69.4 \pm 8.5$ & $165.2 \pm 7.4$ & $54.9 \pm 12.0$ & $20.7 \pm 3.7$ & $76(8.6)$ & $106(12.1)$ & $45(5.1)$ \\
\hline 15 & 1,699 & $112.7 \pm 11.7$ & $69.6 \pm 8.0$ & $170.5 \pm 6.3$ & $61.1 \pm 13.7$ & $21.0 \pm 4.1$ & $177(10.4)$ & $258(15.2)$ & $116(6.8)$ \\
\hline 16 & 1,709 & $114.5 \pm 11.6$ & $71.7 \pm 7.9$ & $172.2 \pm 6.2$ & $63.8 \pm 13.3$ & $21.5 \pm 4.1$ & $212(12.4)$ & $274(16.0)$ & $104(6.1)$ \\
\hline 17 & 560 & $116.0 \pm 10.3$ & $72.7 \pm 8.1$ & $172.9 \pm 5.9$ & $64.5 \pm 13.9$ & $21.6 \pm 4.3$ & $84(15.0)$ & $73(13.0)$ & $37(6.6)$ \\
\hline Total & 25,558 & $105.5 \pm 12.5$ & $66.5 \pm 8.8$ & $145.8 \pm 18.2$ & $41.6 \pm 16.8$ & $18.8 \pm 4.0$ & $2215(8.7)$ & $4570(17.9)$ & $2423(9.5)$ \\
\hline \multicolumn{10}{|l|}{ Girls } \\
\hline 6 & 2,154 & $94.6 \pm 10.1$ & $60.4 \pm 8.3$ & $120.3 \pm 5.2$ & $22.8 \pm 4.4$ & $15.7 \pm 2.4$ & 299(13.9) & $255(11.8)$ & $110(5.1)$ \\
\hline 7 & 2,879 & $97.0 \pm 10.3$ & $62.0 \pm 8.1$ & $125.9 \pm 5.5$ & $25.4 \pm 5.3$ & $16.0 \pm 2.6$ & $436(15.1)$ & $314(10.9)$ & $162(5.6)$ \\
\hline 8 & 2,876 & $99.0 \pm 10.4$ & $63.8 \pm 8.2$ & $131.8 \pm 6.2$ & $28.8 \pm 6.2$ & $16.5 \pm 2.6$ & $367(12.8)$ & $424(14.7)$ & $143(5.0)$ \\
\hline 9 & 2,912 & $101.8 \pm 11.1$ & $65.2 \pm 8.3$ & $137.4 \pm 6.8$ & $32.4 \pm 7.4$ & $17.0 \pm 2.8$ & $382(13.1)$ & $463(15.9)$ & $126(4.3)$ \\
\hline 10 & 2,417 & $104.7 \pm 10.9$ & $66.2 \pm 8.6$ & $144.0 \pm 7.4$ & $37.2 \pm 8.9$ & $17.8 \pm 3.1$ & $271(11.2)$ & $390(16.1)$ & $109(4.5)$ \\
\hline 11 & 725 & $106.3 \pm 11.2$ & $66.6 \pm 8.8$ & $149.5 \pm 7.5$ & $41.6 \pm 10.1$ & $18.4 \pm 3.4$ & $85(11.7)$ & $109(15.0)$ & $30(4.1)$ \\
\hline 12 & 2,587 & $106.6 \pm 11.3$ & $66.7 \pm 8.2$ & $155.6 \pm 6.4$ & $46.7 \pm 10.3$ & $19.2 \pm 3.5$ & $287(11.1)$ & $364(14.1)$ & $111(4.3)$ \\
\hline 13 & 2,643 & $106.9 \pm 11.0$ & $67.5 \pm 8.2$ & $157.7 \pm 5.7$ & $49.3 \pm 9.6$ & $19.7 \pm 3.4$ & $278(10.5)$ & $373(14.1)$ & $72(2.7)$ \\
\hline 14 & 829 & $107.3 \pm 10.7$ & $67.7 \pm 8.1$ & $158.1 \pm 5.8$ & $50.4 \pm 9.2$ & $20.1 \pm 3.3$ & $90(10.9)$ & $76(9.2)$ & $26(3.1)$ \\
\hline 15 & 2,087 & $107.9 \pm 10.6$ & $67.9 \pm 7.6$ & $159.8 \pm 5.5$ & $52.7 \pm 8.9$ & $20.6 \pm 3.2$ & $236(11.3)$ & $203(9.7)$ & $49(2.3)$ \\
\hline 16 & 2,089 & $108.2 \pm 10.9$ & $68.1 \pm 7.7$ & $160.1 \pm 5.6$ & $53.9 \pm 9.9$ & $21.0 \pm 3.5$ & $321(15.4)$ & $222(10.6)$ & $70(3.4)$ \\
\hline 17 & 580 & $108.7 \pm 10.7$ & $68.9 \pm 7.7$ & $160.0 \pm 5.4$ & $54.8 \pm 10.4$ & $21.4 \pm 3.7$ & $93(16.0)$ & $68(11.7)$ & $22(3.8)$ \\
\hline Total & 24,778 & $102.8 \pm 11.6$ & $65.5 \pm 8.5$ & $144.0 \pm 15.4$ & $39.0 \pm 13.8$ & $18.2 \pm 3.6$ & $3145(12.7)$ & $3261(13.2)$ & $1030(4.2)$ \\
\hline
\end{tabular}

Abbreviations: N, sample size; SBP, systolic blood pressure; DBP, diastolic blood pressure; BMI, body mass index.

Notes: Values are mean $\pm \mathrm{SD}$ or $\mathrm{n}(\%)$. 
Table S2. Differences in HBP prevalence among children and adolescents using the Fourth Report and CPG definitions

\begin{tabular}{|c|c|c|c|c|c|}
\hline Age & $\mathrm{N}$ & Fourth Report & $\mathrm{CPG}$ & Difference $^{*}$ & $P^{*}$ \\
\hline \multicolumn{6}{|l|}{ Boys } \\
\hline 6 & 2,277 & $7.2(6.2,8.3)$ & $11.0(9.8,12.4)$ & $3.8(2.2,5.5)$ & $<0.001$ \\
\hline 7 & 3,095 & $9.8(8.7,10.9)$ & $13.3(12.2,14.6)$ & $3 \cdot 6(2 \cdot 0,5 \cdot 2)$ & $<0.001$ \\
\hline 8 & 3,216 & $12.2(11.1,13.4)$ & $18.5(17.2,19.9)$ & $6.3(4.5,8.0)$ & $<0.001$ \\
\hline 9 & 3,168 & $13.3(12.1,14.5)$ & $20.9(19.4,22.3)$ & $7.6(5.7,9.4)$ & $<0.001$ \\
\hline 10 & 2,877 & $12.8(11.6,14.1)$ & $23.4(21.8,25.0)$ & $10.5(8.6,12.5)$ & $<0.001$ \\
\hline 11 & 874 & $12.6(10.5,15.0)$ & $23.3(20.5,26.2)$ & $10.7(7.1,14.3)$ & $<0.001$ \\
\hline 12 & 2,405 & $8.4(7.3,9.6)$ & $18.6(17.0,20.2)$ & $10.2(8.3,12.1)$ & $<0.001$ \\
\hline 13 & 2,799 & $9.6(8.5,10.7)$ & $9.5(8.4,10.7)$ & $-0.1(-1.6,1.5)$ & 0.926 \\
\hline 14 & 879 & $10.4(8.4,12.6)$ & $11.5(9.5,13.8)$ & $1.2(-1.7,4.1)$ & 0.430 \\
\hline 15 & 1,699 & $5.9(4.8,7.1)$ & $10.6(9.2,12.2)$ & $4.7(2.9,6.6)$ & $<0.001$ \\
\hline 16 & 1,709 & $6.8(5.7,8.1)$ & $14.2(12.6,16.0)$ & $7.4(5.3,9.4)$ & $<0.001$ \\
\hline 17 & 560 & $6.3(4.4,8.6)$ & $14.8(12.0,18.0)$ & $8.6(5.0,12.1)$ & $<0.001$ \\
\hline \multicolumn{6}{|l|}{ Girls } \\
\hline 6 & 2,154 & $7.3(6.2,8.5)$ & $9.2(8.0,10.5)$ & $1.9(0.2,3.5)$ & 0.025 \\
\hline 7 & 2,879 & $8.3(7.4,9.4)$ & $10.3(9.2,11.5)$ & $2.0(0.5,3.5)$ & 0.009 \\
\hline 8 & 2,876 & $10.7(9.6,11.9)$ & $13.5(12.3,14.8)$ & $2.8(1.1,4.5)$ & 0.001 \\
\hline 9 & 2,912 & $13.0(11.8,14.3)$ & $17.2(15.9,18.6)$ & $4.2(2.4,6.0)$ & $<0.001$ \\
\hline 10 & 2,417 & $15.7(14.3,17.2)$ & $23.7(22.0,25.4)$ & $8.0(5.8,10.2)$ & $<0.001$ \\
\hline 11 & 725 & $13.8(11.4,16.5)$ & $25.0(21.9,28.3)$ & $11.2(7.1,15.2)$ & $<0.001$ \\
\hline 12 & 2,587 & $8.1(7.1,9.2)$ & $15.1(13.8,16.6)$ & $7.0(5.3,8.8)$ & $<0.001$ \\
\hline 13 & 2,643 & $7.8(6.8,8.9)$ & $5.8(4.9,6.7)$ & $-2.1(-3.4,-0.7)$ & 0.003 \\
\hline 14 & 829 & $6.9(5.2,8.8)$ & $6.2(4.6,8.0)$ & $-0.7(-3.1,1.7)$ & 0.555 \\
\hline 15 & 2,087 & $3.1(2.4,4.0)$ & $3.5(2.8,4.4)$ & $0.4(-0.7,1.5)$ & 0.436 \\
\hline 16 & 2,089 & $2.2(1.6,2.9)$ & $3 \cdot 8(3 \cdot 0,4 \cdot 7)$ & $1.6(0.6,2.7)$ & 0.002 \\
\hline 17 & 580 & $3 \cdot 3(2 \cdot 0,5 \cdot 1)$ & $5.0(3.4,7.1)$ & $1.7(-0.6,4.0)$ & 0.140 \\
\hline
\end{tabular}

Note: *indicate the HBP prevalence gap calculated by using CPG and Fourth Report and examined by Cochran's Q test. 
Table S3. The odds ratios of HBP with BMI groups and height groups in both sexes of two age groups using CPG and the Fourth Report definitions

\begin{tabular}{|c|c|c|c|c|}
\hline \multirow{2}{*}{ Variables } & \multicolumn{2}{|c|}{$6-12$ years } & \multicolumn{2}{|c|}{$13-17$ years } \\
\hline & $\mathrm{CPG}$ & Fourth Report & $\mathrm{CPG}$ & Fourth Report \\
\hline \multicolumn{5}{|l|}{ Boys } \\
\hline \multicolumn{5}{|l|}{ BMI groups } \\
\hline Thinness & $0.58(0.46,0.72)$ & $0.55(0.42,0.73)$ & $0.49(0.29,0.83)$ & $0.46(0.24,0.88)$ \\
\hline Normal & ref & ref & ref & ref \\
\hline Overweight & $2.08(1.86,2.33)$ & $1.97(1.71,2.27)$ & $2.76(2.24,3.39)$ & $2.53(1.99,3.22)$ \\
\hline Obesity & $3.74(3.28,4.26)$ & $3.84(3.29,4.48)$ & $6.26(5.26,7.26)$ & $6.48(5.48,7.48)$ \\
\hline \multicolumn{5}{|l|}{ Height groups } \\
\hline Low & $1.10(0.61,2.01)$ & $1.36(0.74,2.50)$ & $0.56(0.17,1.85)$ & $0.93(0.36,2.36)$ \\
\hline Low-Medium & $1.10(0.89,1.36)$ & $1.25(0.99,1.59)$ & $1.08(0.76,1.53)$ & $1.14(0.78,1.67)$ \\
\hline Medium & ref & ref & ref & ref \\
\hline High-Medium & $1.00(0.86,1.17)$ & $0.89(0.73,1.08)$ & $1.02(0.74,1.39)$ & $1.19(0.84,1.71)$ \\
\hline High & $1.42(1.10,1.83)$ & $1.32(0.96,1.81)$ & $1.21(0.60,2.46)$ & $1.07(0.46,2.48)$ \\
\hline \multicolumn{5}{|l|}{ Girls } \\
\hline \multicolumn{5}{|l|}{ BMI groups } \\
\hline Thinness & $0.63(0.52,0.77)$ & $0.58(0.46,0.73)$ & $0.49(0.26,0.94)$ & $0.61(0.33,1.10)$ \\
\hline Normal & ref & ref & ref & ref \\
\hline Overweight & $1.77(1.56,2.02)$ & $1.79(1.55,2.07)$ & $2.23(1.67,2.98)$ & $2.29(1.71,3.07)$ \\
\hline Obesity & $3.01(2.51,3.61)$ & $3.18(2.60,3.89)$ & $7.62(6.62,8.62)$ & $7.70(6.70,8.70)$ \\
\hline \multicolumn{5}{|l|}{ Height groups } \\
\hline Low & $1.23(0.68,2.21)$ & $1.35(0.73,2.48)$ & $0.98(0.39,2.49)$ & $0.99(0.42,2.34)$ \\
\hline Low-Medium & $1.16(0.92,1.47)$ & $1.12(0.86,1.45)$ & $0.78(0.51,1.19)$ & $0.86(0.57,1.31)$ \\
\hline Medium & ref & ref & ref & ref \\
\hline High-Medium & $1.27(1.08,1.51)$ & $1.15(0.95,1.40)$ & $0.98(0.57,1.71)$ & $0.80(0.43,1.51)$ \\
\hline High & $1.45(1.07,1.97)$ & $1.07(0.74,1.54)$ & $3.07(2.07,4.07)$ & $2.01(1.01,3.01)$ \\
\hline \multicolumn{5}{|l|}{ Both Sexes } \\
\hline \multicolumn{5}{|l|}{ BMI groups } \\
\hline Thinness & $0.60(0.52,0.69)$ & $0.57(0.48,0.68)$ & $0.49(0.32,0.73)$ & $0.53(0.35,0.83)$ \\
\hline Normal & ref & ref & ref & ref \\
\hline Overweight & $1.96(1.80,2.13)$ & $1.87(1.69,2.07)$ & $2.57(2.18,3.04)$ & $2.44(2.03,2.93)$ \\
\hline Obesity & $3.53(3.18,3.92)$ & $3.49(3.09,3.93)$ & $6.46(5.25,7.95)$ & $6.72(5.36,8.43)$ \\
\hline \multicolumn{5}{|l|}{ Height groups } \\
\hline Low & $1.18(0.78,1.79)$ & $1.38(0.90,2.11)$ & $1.53(0.78,2.99)$ & $1.71(0.95,3.06)$ \\
\hline Low-Medium & $1.13(0.97,1.32)$ & $1.19(1.00,1.42)$ & $1.33(1.04,1.71)$ & $1.36(1.05,1.76)$ \\
\hline Medium & ref & ref & ref & ref \\
\hline High-Medium & $1.12(1.00,1.26)$ & $1.00(0.87,1.15)$ & $0.73(0.58,0.93)$ & $0.80(0.61,1.05)$ \\
\hline High & $1.46(1.20,1.78)$ & $1.21(0.96,1.54)$ & $0.84(0.48,1.50)$ & $0.71(0.36,1.39)$ \\
\hline
\end{tabular}

Note: The ORs adjusting for age, regions, rural/urban areas, height (only in BMI groups), birth weight, breastfeeding, delivery ways, gestational age, family hypertension history and household economic level. The ORs in BMI groups and height groups were calculated based on the normal weight group and medium height group respectively after adjusting for the confounders above. 
Table S4. The interaction of odds ratios of HBP with BMI and height in different height groups and BMI groups in both sexes of two age groups using the CPG and Fourth Report definitions

\begin{tabular}{|c|c|c|c|c|}
\hline \multirow{2}{*}{ Variables } & \multicolumn{2}{|c|}{$6-12$ years } & \multicolumn{2}{|c|}{$13-17$ years } \\
\hline & $\mathrm{CPG}$ & Fourth Report & CPG & Fourth Report \\
\hline \multicolumn{5}{|l|}{ Boys } \\
\hline \multicolumn{5}{|c|}{ HBP*Height(in BMI groups) } \\
\hline Thinness & $1.01(0.97,1.05)$ & $1.05(0.95,1.14)$ & $1.01(0.97,1.06)$ & $1.08(0.91,1.21)$ \\
\hline Normal & $1.01(1.00,1.02)$ & $1.20(1.14,1.27)$ & $1.04(1.02,1.06)$ & $1.26(1.16,1.37)$ \\
\hline Overweight & $1.02(1.01,1.04)$ & $1.14(1.03,1.27)$ & $1.04(1.02,1.07)$ & $1.22(1.05,1.41)$ \\
\hline Obesity & $1.01(0.99,1.03)$ & $1.05(1.01,1.10)$ & $1.10(1.06,1.14)$ & $1.15(1.05,1.25)$ \\
\hline \multicolumn{5}{|c|}{ HBP*BMI(in Height groups) } \\
\hline Low & $1.26(1.02,1.54)$ & $1.19(0.98,1.44)$ & $1.30(1.05,1.55)$ & $1.41(1.17,1.58)$ \\
\hline Low-Medium & $1.11(1.02,1.19)$ & $1.14(1.04,1.25)$ & $1.19(1.12,1.27)$ & $1.20(1.13,1.29)$ \\
\hline Medium & $1.17(1.15,1.19)$ & $1.17(1.15,1.20)$ & $1.18(1.15,1.21)$ & $1.17(1.14,1.20)$ \\
\hline High-Medium & $1.18(1.15,1.22)$ & $1.16(1.13,1.20)$ & $1.27(1.20,1.34)$ & $1.26(1.19,1.34)$ \\
\hline High & $1.12(1.08,1.18)$ & $1.13(1.07,1.19)$ & $1.21(1.05,1.39)$ & $1.16(1.02,1.32)$ \\
\hline \multicolumn{5}{|l|}{ Girls } \\
\hline \multicolumn{5}{|c|}{ HBP*Height(in BMI groups) } \\
\hline Thinness & $1.01(0.98,1.04)$ & $1.05(0.93,1.16)$ & $1.01(0.91,1.11)$ & $0.91(0.77,1.11)$ \\
\hline Normal & $1.02(1.01,1.03)$ & $1.11(1.05,1.17)$ & $1.03(1.01,1.06)$ & $1.20(1.09,1.31)$ \\
\hline Overweight & $1.03(1.01,1.04)$ & $1.13(1.01,1.26)$ & $1.03(0.99,1.08)$ & $1.15(0.97,1.36)$ \\
\hline Obesity & $1.03(0.99,1.06)$ & $0.96(0.91,1.02)$ & $1.07(1.00,1.14)$ & $1.17(1.00,1.37)$ \\
\hline \multicolumn{5}{|c|}{ HBP*BMI(in Height groups) } \\
\hline Low & $1.57(1.32,1.82)$ & $1.66(1.40,2.01)$ & $1.35(1.10,1.65)$ & $1.37(1.16,1.61)$ \\
\hline Low-Medium & $1.09(1.02,1.16)$ & $1.07(1.00,1.14)$ & $1.17(1.08,1.27)$ & $1.19(1.10,1.28)$ \\
\hline Medium & $1.13(1.11,1.16)$ & $1.13(1.11,1.16)$ & $1.19(1.15,1.23)$ & $1.20(1.16,1.24)$ \\
\hline High-Medium & $1.11(1.08,1.15)$ & $1.12(1.08,1.16)$ & $1.38(1.21,1.58)$ & $1.34(1.17,1.53)$ \\
\hline High & $1.09(1.03,1.16)$ & $1.15(1.07,1.23)$ & $0.80(0.56,1.15)$ & $0.90(0.66,1.25)$ \\
\hline \multicolumn{5}{|l|}{ Total } \\
\hline \multicolumn{5}{|c|}{ HBP*Height(in BMI groups) } \\
\hline Thinness & $1.01(0.99,1.04)$ & $0.98(0.72,1.32)$ & $1.05(1.01,1.09)$ & $0.81(0.56,1.18)$ \\
\hline Normal & $1.02(1.01,1.02)$ & $1.15(1.11,1.20)$ & $1.06(1.05,1.07)$ & $1.20(1.13,1.28)$ \\
\hline Overweight & $1.02(1.01,1.04)$ & $1.14(1.05,1.23)$ & $1.06(1.04,1.08)$ & $1.16(1.04,1.29)$ \\
\hline Obesity & $1.01(1.00,1.03)$ & $1.02(0.99,1.06)$ & $1.09(1.06,1.11)$ & $1.16(1.08,1.24)$ \\
\hline \multicolumn{5}{|c|}{ HBP*BMI(in Height groups) } \\
\hline Low & $1.27(1.10,1.46)$ & $1.24(1.08,1.43)$ & $1.24(1.09,1.40)$ & $1.27(1.12,1.43)$ \\
\hline Low-Medium & $1.09(1.04,1.15)$ & $1.10(1.05,1.16)$ & $1.18(1.12,1.23)$ & $1.19(1.13,1.25)$ \\
\hline Medium & $1.16(1.14,1.17)$ & $1.15(1.14,1.17)$ & $1.18(1.16,1.20)$ & $1.18(1.16,1.20)$ \\
\hline High-Medium & $1.15(1.13,1.18)$ & $1.13(1.11,1.16)$ & $1.29(1.23,1.35)$ & $1.27(1.21,1.34)$ \\
\hline High & $1.12(1.08,1.16)$ & $1.13(1.09,1.18)$ & $1.11(0.99,1.26)$ & $1.07(0.94,1.21)$ \\
\hline
\end{tabular}

Note: the ORs adjusting for age, regions, rural/urban areas, height (only in BMI groups), birth weight, breastfeeding, delivery ways, gestational age, family hypertension history and household economic level. The ORs of HBP with BMI and height in different height groups and BMI groups were calculated based on continuous variables of BMI and height, respectively. 
Table S5. The prevalence of HBP in different height and BMI groups in both sexes of two age groups using the CPG and Fourth Report definitions, \%

\begin{tabular}{|c|c|c|c|c|c|c|c|}
\hline \multirow{2}{*}{ BMI Groups } & \multirow{2}{*}{ Height Groups } & \multicolumn{2}{|c|}{ 6-12 years } & \multicolumn{2}{|c|}{ 13-17 years } & \multicolumn{2}{|c|}{ Total } \\
\hline & & $\mathrm{CPG}$ & Fourth Report & $\mathrm{CPG}$ & Fourth Report & $\mathrm{CPG}$ & Fourth Report \\
\hline \multicolumn{8}{|l|}{ Boys } \\
\hline & Low & 13.3 & 9.5 & 5.3 & 4.3 & 10.1 & 7.4 \\
\hline \multirow[t]{3}{*}{ Normal } & Medium & 13.1 & 6.0 & 7.6 & 5.2 & 11.4 & 5.8 \\
\hline & High & 14.8 & 5.4 & 10.4 & 9.4 & 13.8 & 6.3 \\
\hline & Low & 24.5 & 15.0 & 19.6 & 20.1 & 21.5 & 18.1 \\
\hline \multirow[t]{2}{*}{ OW\&OB } & Medium & 28.9 & 15.4 & 23.4 & 17.8 & 27.2 & 16.2 \\
\hline & High & 32.7 & 15.3 & 31.5 & 25.5 & 32.5 & 16.7 \\
\hline \multicolumn{8}{|l|}{ Girls } \\
\hline \multirow{4}{*}{ Normal } & Low & 13.3 & 7.4 & 2.8 & 2.9 & 7.5 & 4.9 \\
\hline & Medium & 11.9 & 6.6 & 3.7 & 3.4 & 9.2 & 5.5 \\
\hline & High & 15.4 & 7.2 & 4.4 & 3.3 & 13.8 & 6.6 \\
\hline & Low & 18.4 & 10.5 & 9.2 & 14.1 & 14.1 & 13.3 \\
\hline \multirow[t]{2}{*}{ OW\&OB } & Medium & 25.3 & 15.6 & 11.8 & 13.3 & 20.8 & 14.8 \\
\hline & High & 27.4 & 15.9 & 16.5 & 18.3 & 26.5 & 16.1 \\
\hline \multicolumn{8}{|l|}{ Total } \\
\hline \multirow{4}{*}{ Normal } & Low & 13.3 & 8.5 & 3.7 & 3.4 & 8.6 & 6.0 \\
\hline & Medium & 12.5 & 6.3 & 5.6 & 4.3 & 10.2 & 5.6 \\
\hline & High & 15.1 & 6.3 & 8.1 & 7.0 & 13.8 & 6.5 \\
\hline & Low & 22.0 & 13.1 & 14.8 & 16.4 & 17.1 & 15.4 \\
\hline \multirow[t]{2}{*}{ OW\&OB } & Medium & 27.4 & 15.5 & 18.5 & 15.9 & 24.6 & 15.6 \\
\hline & High & 30.7 & 15.5 & 27.9 & 23.8 & 30.3 & 16.5 \\
\hline
\end{tabular}

Note: OW\&OB, overweight and obesity. Height Groups were classified as a sex-and age-specific height z-score of -1.0 and 1.0 of z-scores into three groups (Low, Medium and High), respectively. 


\begin{tabular}{cccc}
\hline Age, years & Boys & Girls & Total \\
\hline $\mathbf{6}$ & $\mathbf{0 . 2 5}$ & $\mathbf{0 . 2 6}$ & $\mathbf{0 . 2 5}$ \\
$\mathbf{7}$ & $\mathbf{0 . 3 5}$ & $\mathbf{0 . 3 5}$ & $\mathbf{0 . 3 5}$ \\
$\mathbf{8}$ & $\mathbf{0 . 4 1}$ & $\mathbf{0 . 3 2}$ & $\mathbf{0 . 3 7}$ \\
$\mathbf{9}$ & $\mathbf{0 . 2 7}$ & $\mathbf{0 . 2 7}$ \\
$\mathbf{1 0}$ & $\mathbf{0 . 2 5}$ & $\mathbf{0 . 3 1}$ \\
$\mathbf{1 1}$ & $\mathbf{0 . 1 0}$ & $\mathbf{0 . 3 8}$ & $\mathbf{0 . 1 4}$ \\
$\mathbf{1 2}$ & $\mathbf{0 . 3 9}$ & $\mathbf{0 . 1 9}$ & 0.05 \\
13 & 0.28 & $\mathbf{0 . 0 7}$ & -0.33 \\
14 & -0.22 & -0.19 & -0.35 \\
15 & -0.15 & -0.45 & -0.36 \\
16 & -0.32 & -0.51 & -0.42 \\
17 & -0.38 & -0.39 & 0.15 \\
Total & 0.22 & -0.46 & 0.07 \\
\hline
\end{tabular}

Note: the sex- and age-specific height Z-Scores were calculated based on the references in the CDC Growth Charts (www.cdc.gov/growthcharts). 

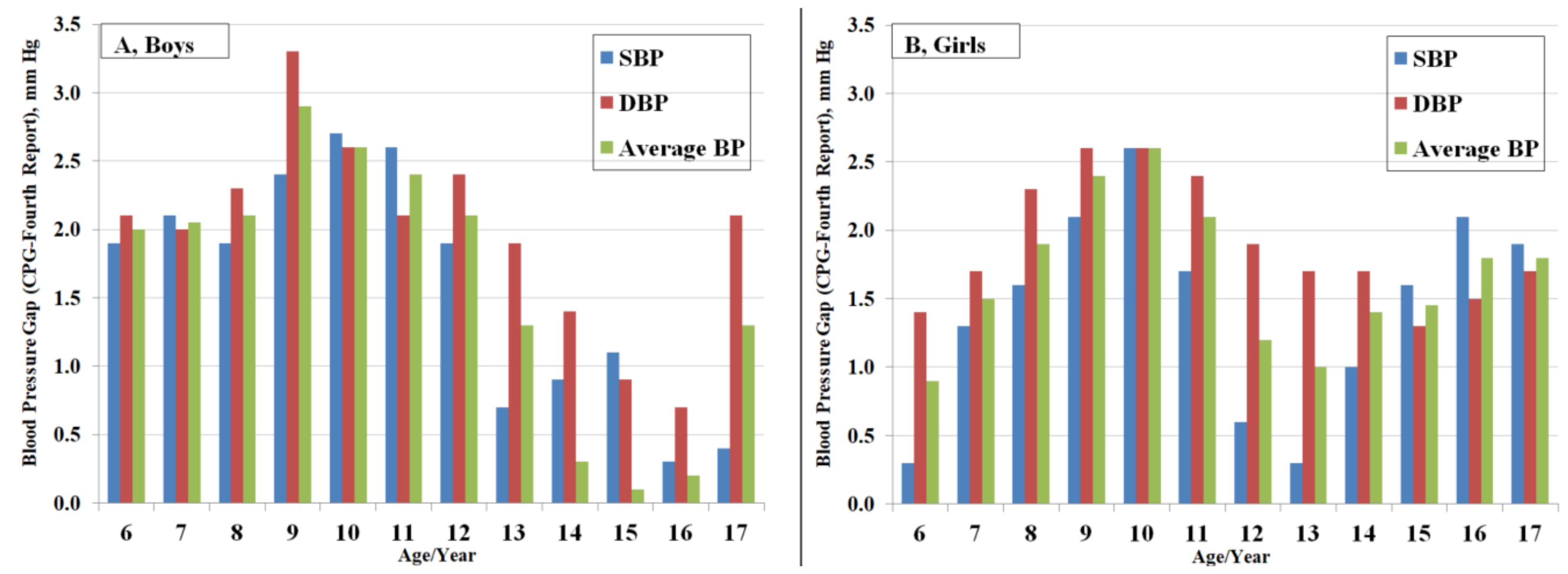

Figure S1. The differences of systolic BP and diastolic BP reference values (P95) between CPG and the Fourth Report across age groups in boys and girls

Note: The age-, sex- and height-specific reference values (P95) of systolic BP (SBP), diastolic BP (DBP) were compared between CPG and the Fourth Report based on the definition tables in two guidelines. The average sex- and age-specific BP reference difference values (Blue bars for SBP; red bars for DBP; green bars for average difference values of SBP and DBP) were presented in the figure. 